\title{
REVIEW
}

\section{Evidence-based treatment strategies in idiopathic pulmonary fibrosis}

\author{
Jürgen Behr
}

ABSTRACT: Recently updated guidelines have provided revised recommendations, based on the GRADE criteria, for the diagnosis and pharmacological and non-pharmacological management of patients with idiopathic pulmonary fibrosis (IPF). Cochrane reviews are also a highly respected and reliable source of evidence-based information that identify and analyse all available data of overall treatment effects from appropriate studies. A recent update of one Cochrane review failed to identify any new evidence supporting the use of corticosteroids in IPF. Another review of data from 15 randomised controlled studies of non-steroid agents for the treatment of IPF identified two trials of interferon- $\gamma-1 b$ suitable for analysis. However, the pooled analysis revealed no treatment effect in terms of survival. A further meta-analysis of three phase III studies of pirfenidone treatment in IPF patients suggested a significant increase in progression-free survival. The interpretation of recent international and national European guideline updates and treatment recommendations, available clinical data from published and ongoing trials investigating potential pharmacological agents, and the individual patient's preferences, must be considered in the clinical management of this disease.

KEYWORDS: Cochrane, evidence based, guidelines, idiopathic pulmonary fibrosis, pirfenidone, treatment recommendations

$\mathbf{T}$ he management and treatment of patients with idiopathic pulmonary fibrosis (IPF) is largely based on the recommendations of prominent societies, such as the American Thoracic Society (ATS) and the European Respiratory Society (ERS). The initial recommendations of the ATS/ERS joint committee were published in 2000 [1]. However, as there were no data to demonstrate any benefit of existing treatment in IPF patients, the guidelines were revised and updated. The 2011 joint statement of the ATS/ERS/Japanese Respiratory Society (JRS)/Latin American Thoracic Association (ALAT) provided further guidance and included mini-reviews of each of the therapeutic agents used in studies for the treatment of patients with IPF [2].

The 2011 ATS/ERS/JRS/ALAT committee guidelines were based on the previously published evidence-based ATS GRADE (Grading of Recommendations, Assessment, Development and Evaluation) rating criteria to determine the quality of available evidence and strength of treatment options [3, 4]. Following a complete review of the literature for each therapeutic agent and assessment of available data, the guidelines provide a combination of either a "Strong" or "Weak" and "Yes" or "No" recommendation for a specific treatment [2]. The strength of a recommendation reflects the extent to which the treating physician can be confident that the beneficial effects of a treatment outweigh the undesirable effects in the range of patients for whom the treatment is intended. Weak No recommendations are a particular challenge as, whilst they imply that such interventions or treatments are not recommended for the majority of patients, they may be of benefit in a significant number of patients with IPF.

Consequently, it is the task and explicit duty of the physician to communicate the potential advantages, and possible disadvantages, of available therapies in order to ascertain the most appropriate management plan that respects the needs and preferences of individual patients.

In addition are, perhaps the most robust assessment of the magnitude of treatment effect, the evidenced-based Cochrane systematic reviews published in the Cochrane Library. The Cochrane Collaboration pioneered the systematic evaluation,
AFFILIATION

Asklepios Fachkliniken München Gauting, Dept of Internal Medicine V Comprehensive Pneumology Center, University of Munich, Munich, Germany

\section{CORRESPONDENCE}

J. Behr

Asklepios Fachkliniken München Gauting, Medizinischen Klinik und Poliklinik V, Comprehensive Pneumology Center Klinikum der Ludwig-MaximiliansUniversität

München Marchioninistr 15 81377 Munich

Germany

E-mail: Juergen.behr@med.unimuenchen.de

Received:

March 042013

Accepted after revision:

April 042013

PROVENANCE

Publication of this peer-reviewed article was supported by InterMune Inc., USA (principal sponsor, European Respiratory Review issue 128). 
analysis and appraisal of all existing and available data from placebo-controlled randomised clinical trials (RCTs) based on pre-defined criteria using the powerful statistical method of meta-analyses $[5,6]$.

\section{NON-PHARMACOLOGICAL TREATMENT}

In terms of non-pharmacological treatment options for IPF patients, long-term oxygen treatment and lung transplantation received Strong Yes recommendations from the 2011 ATS/ERS/ JRS/ALAT committee guidelines [2]. Pulmonary rehabilitation was given a Weak Yes recommendation. There were Weak No recommendations for specific treatment for pulmonary hypertension and for mechanical ventilation for respiratory failure due to IPF.

\section{PHARMACOLOGICAL TREATMENT}

\section{Anticoagulation therapy}

The use of anticoagulants administered with or without corticosteroids was initially investigated in a small, randomised, open-label study in Japan [7]. A survival benefit $(p=0.04)$ was demonstrated in patients treated with anticoagulation therapy $(n=23)$ compared with no anticoagulation $(n=33)$. This was thought to be due predominantly to reduced hospitalisations for acute exacerbation and/or worsening respiratory function. However, there were significant limitations to this study including the absence of blinding, imbalanced dropout rates, failure to exclude pulmonary embolism as the cause of deterioration, and insufficient documentation regarding anticoagulant administration during outpatient treatment. Therefore, based on the available evidence anticoagulant therapy received a Weak No recommendation from the ATS/ ERS/JRS/ALAT committee [2].

In the later US government-funded ACE-IPF (AntiCoagulant Effectiveness in Idiopathic Pulmonary Fibrosis) study, warfarin (coumadin) was associated with an increased risk of mortality in an IPF population who lacked other indications for anticoagulation [8]. This study ended in 2011 due to ineffectiveness after 145 of the planned 256 subjects were enrolled. Based on this new evidence, the more recent German IPF guideline placed a Strong No recommendation to warfarinbased anticoagulation in IPF [9].

\section{Anti-inflammatory or immunosuppressive therapies}

Cochrane reviews have consistently shown a paucity of appropriate data from RCTs assessing the efficacy of corticosteroid therapy in IPF patients for inclusion in a systematic review [10]. Although the evidence to support the routine use of corticosteroids in IPF is weak, there are no data to completely exclude their use in other forms of pulmonary fibrosis. Thus, and in the absence of alternative therapy, corticosteroids may be considered a reasonable option in some patients such as those with nonspecific interstitial pneumonia or with acute exacerbation(s) of IPF [2]. It was concluded that an accurate diagnosis in each patient is crucial, while the benefit of immunomodulatory and anti-inflammatory or immunosuppressive therapies for the effective treatment of IPF remains to be proven [10].

\section{Combination therapy}

The IFIGENIA (Idiopathic Pulmonary Fibrosis International Group Exploring N-Acetylcysteine I Annual) study $(\mathrm{n}=155)$ investigated high-dose $N$-acetylcysteine (NAC; $600 \mathrm{mg}$ three times daily) versus placebo on a "standard" background therapy of prednisone and azathioprine [11]. NAC was found to significantly reduce decline in the primary end-point of change in vital capacity and diffusing capacity of the lung for carbon monoxide after 1 year $(p \geqslant 0.02)$. However, there were numerous limitations to interpreting these data, as not all patients were included in the intention-to-treat analysis. In addition, use of the last observation carried forward method of analysis, which inflates Type I errors, may overestimate treatment effect. It was also unclear how important the observed effect was with regard to patient relevant outcomes including quality of life, dyspnoea and survival. Finally, there is also an ongoing debate as to whether the observed treatment effect could be attributed to either NAC alone or the combination of NAC with prednisone and azathioprine (socalled triple therapy) [12]. Considering these obvious limitations, both triple therapy and NAC monotherapy received a Weak No recommendation [2].

In an attempt to clarify this ongoing dispute, the US government sponsored the PANTHER-IPF (Prednisone, Azathioprine, and $N$-Acetylcysteine: A Study That Evaluates Response in Idiopathic Pulmonary Fibrosis) study which compared three treatment arms: triple therapy, NAC monotherapy, and placebo for all three substances. The recently published results of this study demonstrated that there was an increased risk of death and hospitalisations in patients with IPF treated with triple therapy [13]. The precise reasons for the increased rates of death and hospitalisation are unknown and it is difficult to assess which components of the three-drug regimen may be responsible for the observed negative outcomes [14]. Consequently, the triple-therapy arm of the PANTHER-IPF study was terminated early, although as the use of NAC anti-oxidant monotherapy remains inconclusive, the NAC monotherapy and placebo arms of the study are continuing. The ongoing comparison of NAC alone and matching placebo will address the therapeutic role of NAC alone for patients with IPF.

\section{IMMUNOMODULATORS}

There is little quality data regarding the efficacy of new noncorticosteroid immunomodulatory agents in patients with IPF. A Cochrane review identified 59 RCTs of nonsteroid agents for the treatment of IPF; however, the quality of these studies was generally poor and only three were suitable for meta-analyses [15]. In 2010, a further Cochrane review identified 13 suitable RCTs together with two additional ongoing RCTs due to be published soon after the analysis, thus allowing their inclusion in discussion [16]. Due to the analysis of the end-points and the quality of the methodology eight trials were omitted and only seven were eligible for meta-analyses. Only the anti-fibrotic agents interferon (IFN)- $\gamma-1 b$ and pirfenidone were evaluated in more than one trial and were, therefore, potentially eligible for two separate meta-analyses.

\section{Interferon- $\gamma-\mathbf{1 b}$}

Two placebo-controlled RCTs of IFN- $\gamma-1 b$ therapy have been conducted in patients with IPF. The first and smaller of the two 
trials $(n=330)$ almost demonstrated a significantly greater effect of IFN- $\gamma-1 b$ compared with placebo treatment in IPF patients in terms of overall survival $(p=0.08)$, but not in the primary endpoint of improved progression-free survival (PFS) [17]. Data from the subsequent and larger INSPIRE (A Randomised, Double-Blind, Placebo-Controlled, Phase 3 Study of the Safety and Efficacy of Interferon Gamma-1b in Patients With Idiopathic Pulmonary Fibrosis) study $(\mathrm{n}=826)$ also failed to show a survival benefit in IPF patients treated with IFN- $\gamma-1 b$ [18].

The Cochrane meta-analysis combining the data from the two IFN- $\gamma-1 b$ RCTs showed no significant difference in the clinical end-point of overall survival between IFN- $\gamma-1 \mathrm{~b}$ and placebo $[15,16]$. IFN- $\gamma-1 b$ therefore received a Strong No recommendation from the ATS/ERS/JRS/ALAT committee, largely based on the high value placed on treatment cost versus potential risks [2].

\section{Pirfenidone}

Pirfenidone is a small, orally available molecule that demonstrates anti-inflammatory and anti-fibrotic effects in vitro and in vivo [19]. Although no specific mode of action has been identified, treatment-related reductions in fibrosis are associated with modulation of cytokines and growth factors. To date, four placebo-controlled RCTs have evaluated the treatment of IPF patients with pirfenidone. The phase II study $(n=107)$ was stopped early due to the incidence of acute exacerbations in placebo-treated patients, while none occurred with pirfenidone treatment [20]. A Japanese phase III study (SP3) demonstrated a significant effect on decline of forced vital capacity (FVC) and PFS in 267 patients treated with pirfenidone compared with placebo [21].

Pirfenidone in IPF patients was further investigated in the CAPACITY (Clinical Studies Assessing Pirfenidone in IPF: Research of Efficacy and Safety Outcomes) study consisting of two concurrent multi-national RCTs (studies 004 and 006) [22]. While the primary end-point (change in FVC \% predicted from baseline to week 72) was met in study $004(n=435 ; p=0.001)$, it was not met in study $006(n=344)$. However, a significant pirfenidone treatment effect (estimated by differences in treatment group means and categorical change in FVC) was noted all time-points in study $006(\mathrm{p}=0.005)$, and also in a

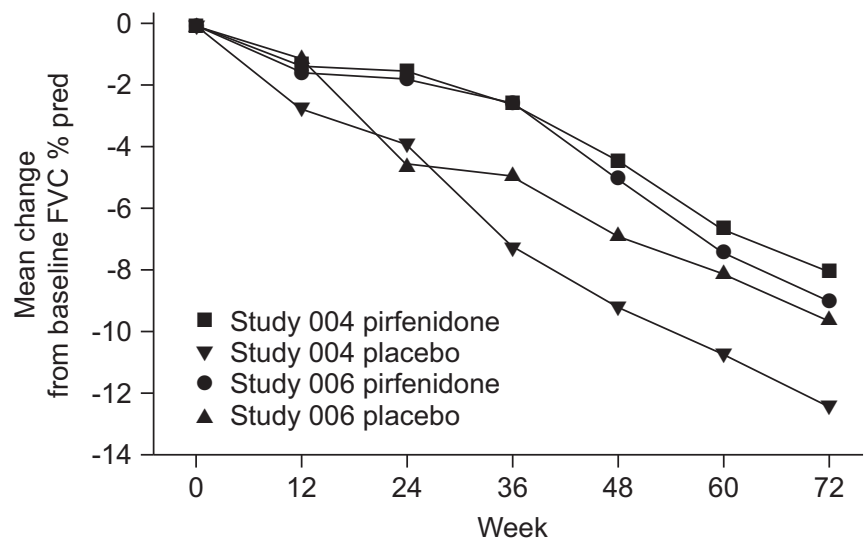

FIGURE 1. Change in forced vital capacity (FVC) \% predicted (\% pred) per week from baseline in the CAPACITY 004 and 006 study comparing pirfenidone (2403 mg.day ${ }^{-1}$ ) or placebo in patients with idiopathic pulmonary fibrosis (IPF). CAPACITY: Clinical Studies Assessing Pirfenidone in IPF: Research of Efficacy and Safety Outcomes. Reproduced from [23]

repeated-measures analysis of FVC \% pred change across all time-points in both studies ( $p=0.0007)$ (fig. 1 and table 1) [23, 24].

The difference in FVC outcomes in the two studies might be partly attributable to a lower than expected rate of FVC decline in the placebo arm of study 006 after 1 year, while the magnitude of decline over time was similar in the two pirfenidone groups. In the primary analyses of both studies, the magnitude of treatment effect was similar at all assessment time-points over 1 year. Indeed, pooled data from both studies provide compelling evidence that pirfenidone reduces decline in lung function and disease progression [22]. At week 72, both studies favoured pirfenidone with overlapping confidence intervals. In addition, a favourable trend for all-cause mortality and mortality related to IPF supports a beneficial treatment effect of pirfenidone in patients with IPF (table 2) [22].

An extension phase of the CAPACITY studies (called RECAP) was designed to assess the safety of pirfenidone beyond the duration of the phase III studies. At week 72 of the RECAP extension study, patients had been treated with pirfenidone for a mean duration of 2.9 years (range $1-4$ years). 114 patients

TABLE 1 Change in forced vital capacity \% predicted per week in the CAPACITY 004 and 006 study

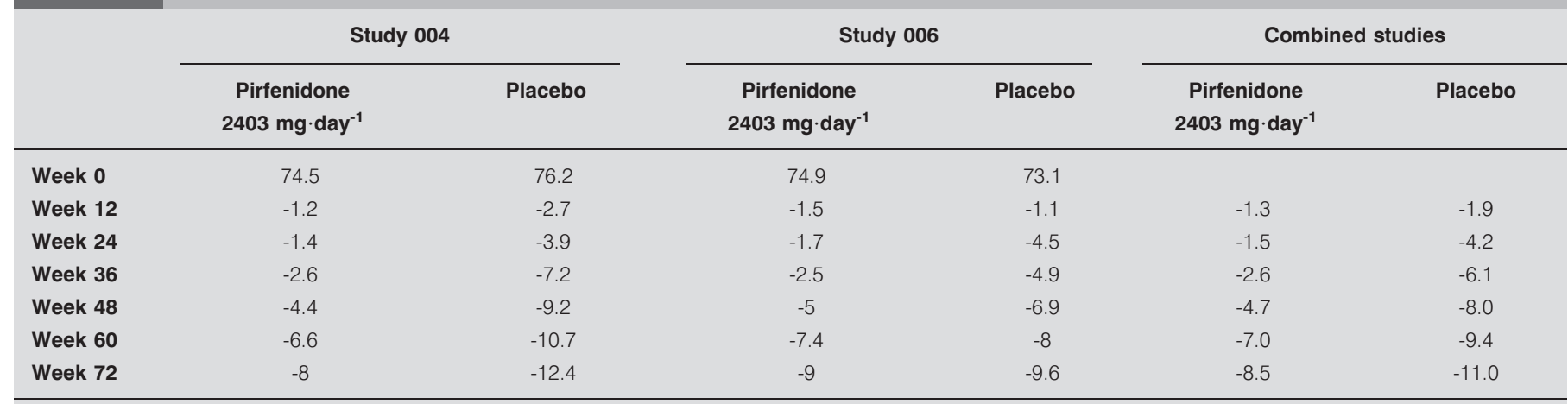

CAPACITY: Clinical Studies Assessing Pirfenidone in IPF: Research of Efficacy and Safety Outcomes. Data from [24]. 


\begin{tabular}{|c|c|c|c|c|}
\hline All-cause mortality & $27(8)$ & $34(10)$ & $0.77(0.47-1.28)$ & 0.315 \\
\hline IPF-related mortality ${ }^{f}$ & $18(5)$ & $28(8)$ & $0.62(0.35-1.13)$ & 0.117 \\
\hline \multicolumn{5}{|l|}{ On-treatment ${ }^{\# \#}$} \\
\hline IPF-related mortality ${ }^{f}$ & $12(3)$ & $25(7)$ & $0.48(0.24-0.95)$ & 0.030 \\
\hline
\end{tabular}

had been treated with the full dose for at least 3 years. Data from the RECAP extension study confirm the tolerability of pirfenidone [25].

The Cochrane meta-analysis of pirfenidone included three clinical trials eligible for analysis, i.e. the Japanese SP3 trial [21] and the two large, international CAPACITY (004 and 006) trials [22]. Although these studies did not have a common primary end-point, PFS data was used as a secondary end-point in both the Japanese and CAPACITY studies. The overall result of this meta-analysis showed that treatment with pirfenidone reduced the risk of disease progression by $30 \%$ (HR $0.70,95 \%$ CI $0.56-$ 0.88) (fig. 2) [16]. Thus, pirfenidone is the only drug to date to have shown a significant effect on PFS compared with placebo in patients with IPF.

While the US Food and Drug Administration (FDA) refused approval of pirfenidone based on the two CAPACITY studies, it was approved for use in Japan and India in 2010, and also in Europe in 2011 by the European Medicines Agency (EMA) for patients with mild-to-moderate IPF based on all available data, including the Japanese studies and the Cochrane review. However, the ATS/ERS/JRS/ALAT guideline committee gave a Weak No recommendation for pirfenidone, with high value placed on costs and side-effects and low value on the possible

Study

HR

IV, Random, 95\% CI

IV, Random, 95\% Cl

CAPACITY 1 [22] $0.84(0.58-1.22)$

CAPACITY 2 [22] $0.64(0.43-0.94)$

TANIGUCHI [21] $0.64(0.43-0.94)$

Total

$0.70(0.56-0.88)$
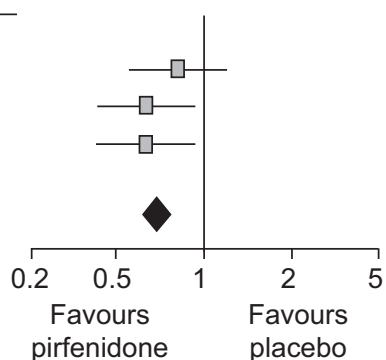

FIGURE 2. Meta-analysis of progression-free survival with pirfenidone versus placebo in idiopathic pulmonary fibrosis (IPF). CAPACITY: Clinical Studies Assessing Pirfenidone in IPF: Research of Efficacy and Safety Outcomes. Reproduced from [16] with permission from the publisher. small reduction in pulmonary decline [2]. It must be noted, however, that the majority of committee members (16 out of 31) abstained from voting on pirfenidone as most were involved in the CAPACITY trials. In addition, the guidelines were devised before full publication of the CAPACITY study data and without taking into consideration the positive findings of the Cochrane meta-analysis. The complete dataset available to date is likely to have an important impact on confidence in the treatment effect of pirfenidone in the future.

\section{IMPLICATIONS FOR CLINICAL PRACTICE}

It is clear that treatment decisions and the clinical management of patients with IPF should be based primarily on the collective findings from RCTs and also, to a certain extent, from expert opinion. However, the ATS/ERS/JRS/ALAT recommendations are based on the GRADE system, which is not understood universally and may not be ideally suited to such a rare condition as IPF that has no established current recommended treatment. Indeed, the discrepancies between the decisions of the FDA, the EMA and the 2011 international guideline

\section{TABLE 3 Summary of evidenced-based treatments for idiopathic pulmonary fibrosis}

\begin{tabular}{|c|c|c|}
\hline & $\begin{array}{l}\text { ATS/ERS/JRS/ALAT } \\
\text { guidelines [2] }\end{array}$ & New evidence 2012 \\
\hline Anticoagulation & Weak No & Strong No (ACE) $[8,9]$ \\
\hline NAC monotherapy & Weak No & Weak No $[2,11]$ \\
\hline $\begin{array}{l}\text { Prednisone/ } \\
\text { azathioprine/NAC }\end{array}$ & Weak No & $\begin{array}{c}\text { Strong No } \\
\text { (PANTHER) }[9,13]\end{array}$ \\
\hline Pirfenidone & Weak No & $\begin{array}{c}\text { Weak Yes [22] } \\
\text { (German guidelines [9]) }\end{array}$ \\
\hline \multicolumn{3}{|c|}{$\begin{array}{l}\text { For definitions of Weak No, Strong No and Weak Yes refer to [2]. ATS: American } \\
\text { Thoracic Society; ERS: European Respiratory Society; JRS: Japanese } \\
\text { Respiratory Society; ALAT: Latin American Thoracic Association; NAC: N- } \\
\text { acetylcysteine; ACE: angiotensin converting enzyme; PANTHER: Prednisone, } \\
\text { Azathioprine, and N-Acetylcysteine: A Study That Evaluates Response in } \\
\text { Idiopathic Pulmonary Fibrosis. }\end{array}$} \\
\hline
\end{tabular}


committee [2] demonstrate that there are different ways to interpret data from RCTs.

Based on the available evidence in 2010, the key message from the 2011 ATS/ERS/JRS/ALAT guidelines is unfortunately that no pharmacological treatments are strongly recommended for patients with IPF (table 3). This is due predominantly to the insufficient or inadequate quality of data regarding the risks and benefits supporting their use [2]. In view of the recently published US government-sponsored clinical trials (ACE-IPF and PANTHER-IPF), the formerly widely accepted view that patients with IPF should be treated with a combined therapy of corticosteroids and an immunosuppressive agent (e.g. azathioprine) plus high-dose oral NAC is no longer appropriate. As a consequence, in countries in which pirfenidone is approved, eligible patients are treated with this new drug for the treatment of mild-to-moderate IPF. NAC monotherapy remains as second-line therapy, at least until the results of the PANTHER NAC versus placebo results are available, which will either prove or disprove the efficacy of NAC.

Individual national European guidelines have been, or are being, updated. For example, the German Health System recently implemented revised, evidence-based guidelines following a review of the international guidelines and other recent publications by German IPF experts at a consensus meeting in December 2011 [9]. Most of the international guideline recommendations were considered applicable to the German IPF population. Recommendations for anticoagulation and combination therapy with prednisone, azathioprine and NAC for patients with definite IPF were changed from a Weak No to a Strong No. Pirfenidone treatment was upgraded from a Weak No to a Weak Yes based on publication of the CAPACITY and Japanese studies showing significant effect on PFS, the Cochrane meta-analysis, new data of the minimal clinically important difference for FVC and 6-min walk test and favourable observed mortality data, as well as an acceptable tolerability profile $[2,16,21,22,25,26]$.

\section{DISCUSSION}

Since 2004 there has been an increase in the number of clinical trials investigating the treatment of IPF, and this is expected to continue. These trials include the use of nine different potential therapeutic agents acting on various different targets and the use of multiple different drugs focusing on trying to find an effective treatment for this disease. As the number of clinical trials completed increases, the question now is how best to analyse the data from these studies to determine a treatment effect. There is clearly an unmet need for an accurate primary end-point for use in clinical trials of treatments for IPF. A European-wide consensus exists that states mortality is not a practical primary end-point for the demonstration of beneficial treatment effects in IPF [13]. FVC change thresholds have important theoretical advantages and are now the preferred primary end-point in IPF treatment trials, although they are not a proven surrogate for mortality [1, 2].

The role of the clinician is, therefore, of the utmost importance in helping the patient to make an informed treatment decision. The implication for the clinician is that most patients should receive a specific intervention if given a strong recommendation. Moreover, a strong recommendation for the use of an intervention should be adopted by policy makers at a given hospital or institution. However, if a patient is well informed, pharmacological treatment with Weak No recommendations should be considered.

Fully informed patients are best placed to make decisions that are consistent with the best available evidence. Physicians must discuss the available options with individual patients and make a treatment decision with the patient, based on their specific values and preferences.

\section{STATEMENT OF INTEREST}

Conflict of interest information can be found alongside the online version of this article at err.ersjournals.com

\section{ACKNOWLEDGEMENTS}

I would like to thank C. Trenam and M. Smith (IntraMed Europe, Milan, Italy) for editorial assistance in the preparation of the manuscript.

\section{REFERENCES}

1 American Thoracic Society. Idiopathic pulmonary fibrosis: diagnosis and treatment. International consensus statement. American thoracic society (ATS), and the European Respiratory Society (ERS). Am J Respir Crit Care Med 2000; 161: 646-664.

2 Raghu G, Collard HR, Egan JJ, et al. An Official ATS/ERS/JRS/ ALAT statement: idiopathic pulmonary fibrosis: evidence-based guidelines for diagnosis and management. Am J Respir Crit Care Med 2011; 183: 788-824.

3 Guyatt G, Gutterman D, Baumann MH, et al. Grading strength of recommendations and quality of evidence in clinical guidelines: report from an American College of Chest Physicians Task Force. Chest 2006; 129: 174-181.

4 Schünemann HJ, Jaeschke R, Cook DJ, et al. Documents Development and Implementation Committee. An official ATS statement: grading the quality of evidence and strength of recommendations in ATS guidelines and recommendations. Am J Respr Crit Care Med 2006; 174: 605-614.

5 Gay SE, Kazerooni EA, Toews GB, et al. Idiopathic pulmonary fibrosis: predicting response to therapy and survival. Am J Respir Crit Care Med 1998; 157: 1063-1072.

6 Mulrow CD. Rationale for systematic reviews. BMJ 1994; 309: 597-599.

7 Kubo H, Nakayama K, Yanai M, et al. Anticoagulant therapy for idiopathic pulmonary fibrosis. Chest 2005; 128: 1475-1482.

8 Noth I, Anstrom KJ, Calvert SB, et al. Idiopathic Pulmonary Fibrosis Clinical Research Network (IPFnet). A placebo-controlled randomized trial of warfarin in idiopathic pulmonary fibrosis. Am J Respir Crit Care Med 2012; 186: 88-95.

9 Behr J, Günther A, Ammenwerth W, et al. S2K-Leitlinie zur Diagnostik und Therapie der idiopathischen Lungenfibrose. [German guideline for diagnosis and management of idiopathic pulmonary fibrosis.]. Pneumologie 2013; 67: 81-111.

10 Richeldi L, Davies HR, Ferrara G, et al. Corticosteroids for idiopathic pulmonary fibrosis. Cochrane Database Syst Rev 2003; 3: CD002880.

11 Demedts M, Behr J, Buhl R, et al. High-dose acetylcysteine in idiopathic pulmonary fibrosis. N Engl J Med 2005; 353: 2229-2242.

12 Hunninghake GW. Antioxidant therapy for idiopathic pulmonary fibrosis. N Engl J Med 2005; 353: 2285-2287.

13 Idiopathic Pulmonary Fibrosis Clinical Research Network. Prednisone, azathioprine, and $\mathrm{N}$-acetylcysteine for pulmonary fibrosis. N Engl J Med 2012; 366: 1968-1977.

14 Behr J. Prednisone, azathioprine an $\mathrm{N}$-acetylcysteine for pulmonary fibrosis. N Engl J Med 2012; 367: 869. 
15 Davies HR, Richeldi L, Walters EH. Immunomodulatory agents for idiopathic pulmonary fibrosis. Cochrane Database Syst Rev 2003; 3: CD003134.

16 Spagnolo P, Del Giovane C, Luppi F, et al. Non-steroid agents for idiopathic pulmonary fibrosis. Cochrane Database Syst Rev 2010; 9: CD003134.

17 Raghu G, Brown KK, Bradford WZ, et al. A placebo-controlled trial of interferon $\gamma-1 \mathrm{~b}$ in patients with idiopathic pulmonary fibrosis. N Engl J Med 2004; 350: 125-133.

18 King TE Jr, Albera C, Bradford WZ, et al. Effect of interferon $\gamma-1 b$ on survival in patients with idiopathic pulmonary fibrosis (INSPIRE): a multicentre, randomised, placebo-controlled trial. Lancet 2009; 374: 222-228.

19 Schaefer CJ, Ruhrmund DW, Pan L, et al. Antifibrotic activities of pirfenidone in animal models. Eur Respir Rev 2011; 20: 85-97.

20 Azuma A, Nukiwa T, Tsuboi E, et al. Double-blind, placebocontrolled trial of pirfenidone in patients with idiopathic pulmonary fibrosis. Am J Respi Crit Care Med 2005; 171: 1040-1047.
21 Taniguchi H, Ebina M, Kondoh Y, et al. Pirfenidone in idiopathic pulmonary fibrosis. Eur Respir J 2010; 35: 821-829.

22 Noble PW, Albera C, Bradford WZ, et al. Pirfenidone in patients with idiopathic pulmonary fibrosis (CAPACITY): two randomised trials. Lancet 2011; 377: 1760-1769.

23 Costabel U. Emerging potential treatments: new hope for idiopathic pulmonary fibrosis patients? Eur Respir Rev 2011; 20: 201-207.

24 European Medicines Agency. EPAR Esbriet. www.ema.europa.eu /ema/index.jsp?curl=pages $/$ medicines $/$ human $/$ medicines $/ 002154$ / human_med_001417.jsp\&mid=WC0b01ac058001d124 Date last updated: January 14, 2013. Date last accessed: April 25, 2013.

25 Costabel U, Albera C, Cohen A, et al. The long-term safety of pirfenidone in patients with idiopathic pulmonary fibrosis (IPF): Interim data from the RECAP extension study. Eur Respir J 2011; 38: Suppl. 55, 35 .

26 Cottin V. Changing the idiopathic pulmonary fibrosis treatment approach and improving patient outcomes. Eur Respir Rev 2012; 21: 161-167. 